\title{
August 2018 Pulmonary Case of the Month
}

\author{
Arooj Kayani, MD \\ Richard Sue, MD \\ Banner University Medical Center Phoenix \\ Phoenix, AZ USA
}

\section{History of Present IIIness}

A 59-year-old woman referred because of worsening dyspnea over the past 2 months along with cough and wheezing. She has a history of chronic obstructive pulmonary disease (COPD) and is on continuous oxygen @ 2 L/min.

\section{$\mathrm{PMH}, \mathrm{SH}$, and $\mathrm{FH}$}

In addition to her COPD she has a history of hypothyroidism, pneumonia, tonsillectomy, hip lipoma resection, hysterectomy, and a herniorrhaphy. She has a 30 pack-year history of smoking. She currently smokes half pack/day. No family history of lung disease or cancer.

\section{Medications}

- Fluticasone/salmeterol

- Tiotropium

- Albuterol

- Levothyroxine

\section{Physical Examination}

- Vitals: HR 79/min, BP 100/69 mmHg, RR 16/min, SpO2 92\% on 2 L/min.

- General: Alert and oriented. Healthy appearing in no distress.

- Lungs: Expiratory stridor and expiratory wheezing loudest over left lung. No crackles.

- Cardiac: Regular rhythm with no murmurs. No edema.

- The remainder of physical examination was unremarkable.

Which of the following should be performed?

1. Spirometry

2. Sputum Gram stain, AFB stain, and fungal stain with cultures

3. Thoracic CT scan

4. 1 and 3

5. All of the above 


\section{Correct! \\ 5. All of the above}

Sputum stains and cultures, spirometry and thoracic CT scan may all be useful in evaluating dyspnea. In this case the least useful would probably be sputum stains and cultures all of which were negative.

Spirometry was performed with the forced vital capacity $2.3 \mathrm{~L}$ and the forced expiratory volume in 1 second $0.55 \mathrm{~L}$ which was unchanged compared to her most recent previous spirometry. The flow volume loop is shown in Figure 1.

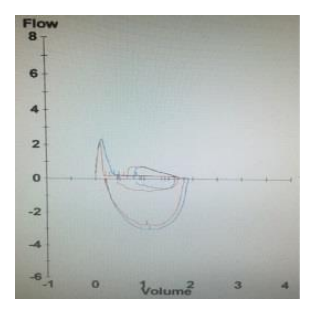

Figure 1. Flow-volume loop consistent with severe lower airway obstruction.

Her thoracic CT scan is shown in Figure 2.

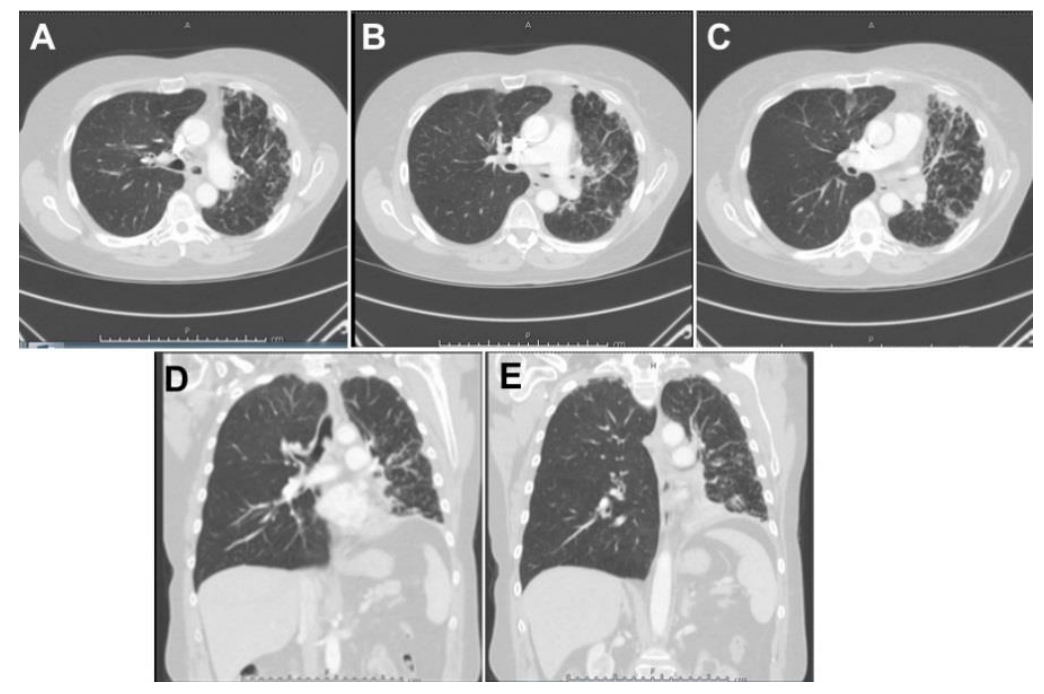

Figure 2. Representative images from initial thoracic CT scan. A-C: axial view in lung windows. $D$ \&E: coronal view in lung windows.

Which of the following should be done next?

1. Antibiotics and prednisone for an exacerbation of COPD

2. Bronchoscopy

3. Pulmonary CT angiogram

4. 1 and 3

5. All of the above 


\section{Correct! \\ 2. Bronchoscopy}

There is little to suggest pulmonary emboli and a pulmonary CT angiogram will be low yield. It seems unlikely that antibiotics and prednisone her dyspnea especially given the CT findings. The best choice is bronchoscopy. The thoracic CT scan shows narrowing of the left mainstem bronchus and left lung volume loss suggesting a mass.

Bronchoscopy was performed and shows a mass in the left mainstem bronchus (Figure $3)$.

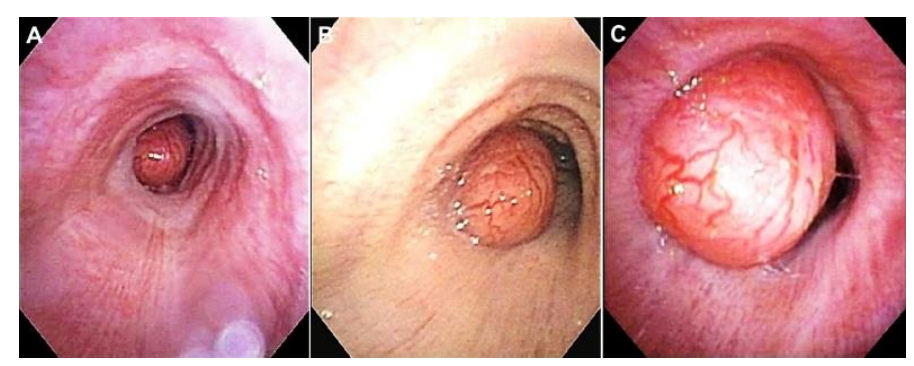

Figure 3. Bronchoscopy beginning just below carina $(A)$ and proceeding towards the mass.

Biopsy of the lesion is shown in Figure 4.

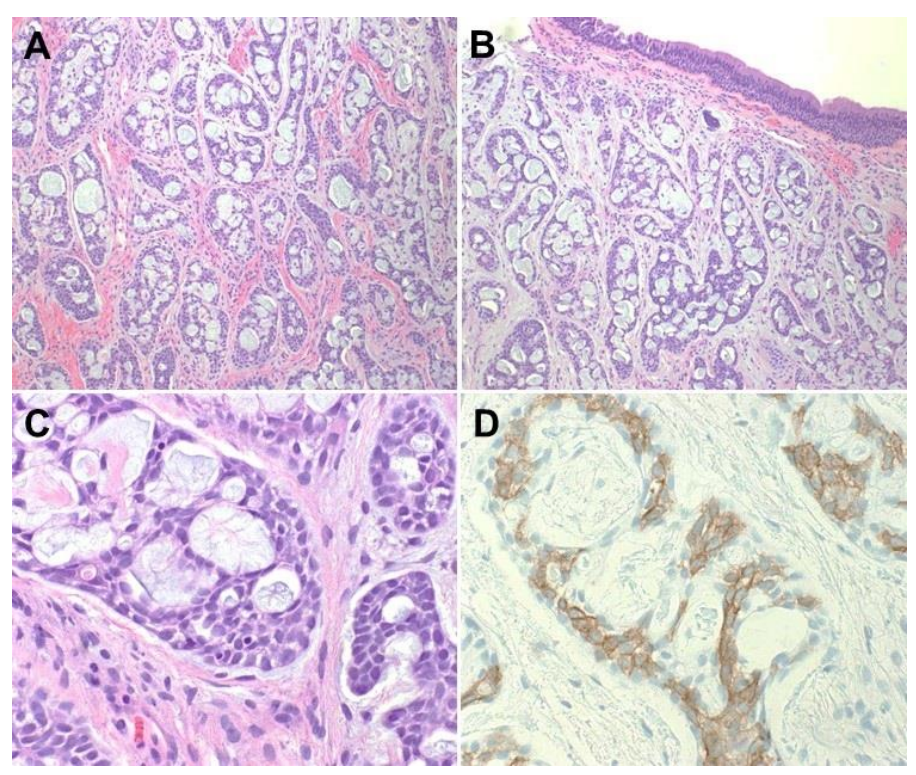

Figure 4. Histopathology of left airway mass. A \& B: H \& E staining under low power. C: $\mathrm{H} \& \mathrm{E}$ under high power. D: High power showing positive immunohistology staining for CD-117 and SOX10.

The histopathology was consistent with adenoid cystic carcinoma $(1,2)$. 
What should be done next?

1. Debulking with rigid bronchoscopy

2. Left pneumonectomy

3. Debulking with laser bronchoscopy

4. 1 or 3

5. Any of the above 


\section{Correct!}

\section{1 or 3}

Adenoid cystic carcinoma (ACC) was previously called a cylindroma $(1,2)$. It is a rare tumor which originates from submucosal glands and can arise where submucosal glands are present. When present in the lung ACC usually arises centrally within large airways as an intraluminal mass, rarely forming a peripheral nodule. It is a slow-growing low-grade malignancy. Symptoms depend on location, often causing cough, dyspnea, wheezing and stridor. Hemoptysis is rare.

The preferred treatment is surgical resection (2). However, our patient has severe oxygen-dependent COPD which precludes a pneumonectomy or sleeve resection. In the case of an unresectable tumor, debulking and radiation therapy are usually performed. Five-year survival ranges between approximately 50 and $80 \%$ in patients with resectable disease, and $30 \%$ for those with unresectable disease. Recurrences can occur as late as 10-15 years after surgical resection. Chemotherapy has not been demonstrated to increase survival.

Our patient underwent debulking initially with a snare to remove large segments and biopsy forceps by rigid bronchoscopy. KTP laser was used to remove fragments. KTP laser to remove remnants (Figure 5).

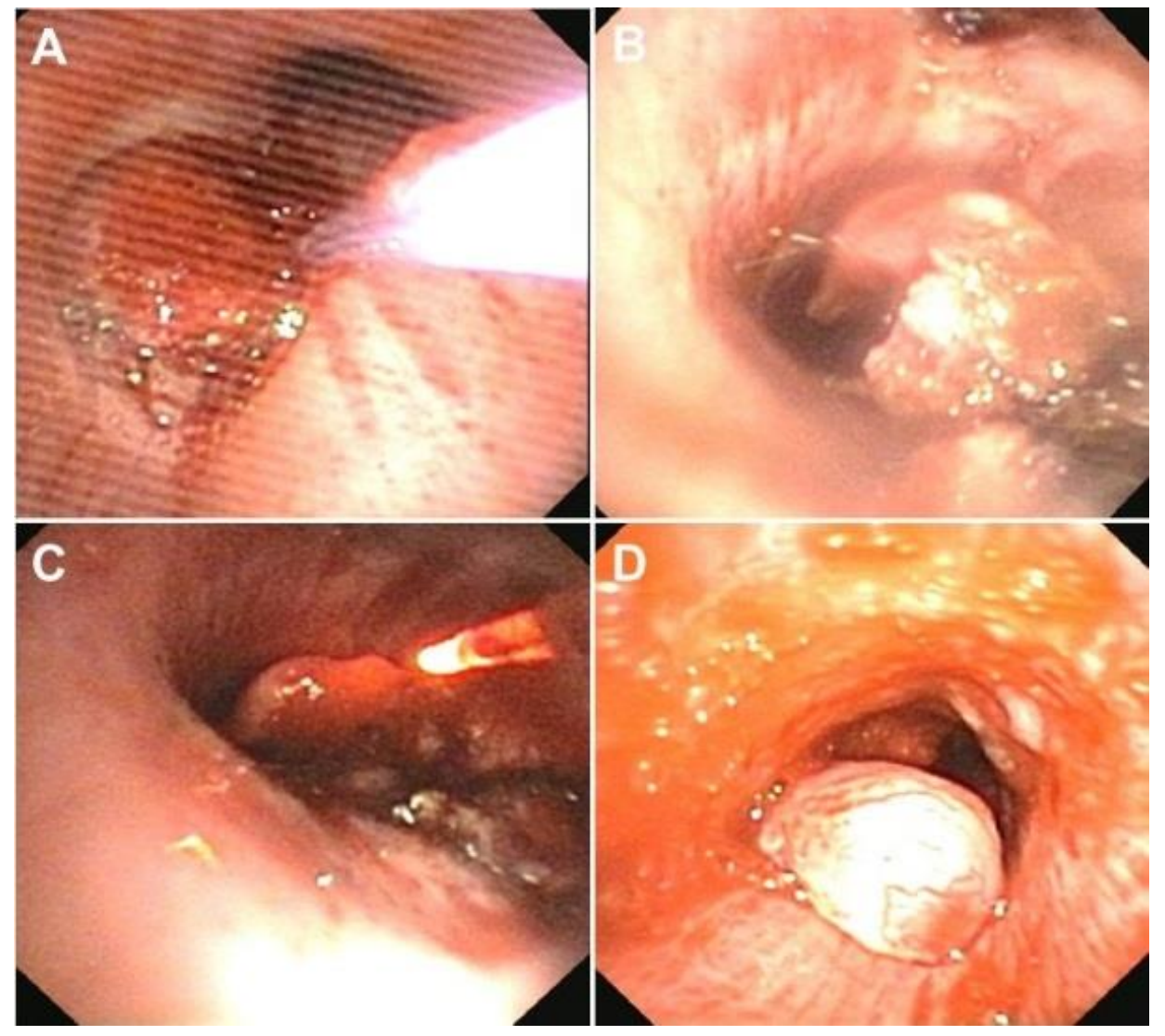

Figure 5. Initial KTP laser. 
A second session was performed with the KTP laser 4 months later (Figure 6).

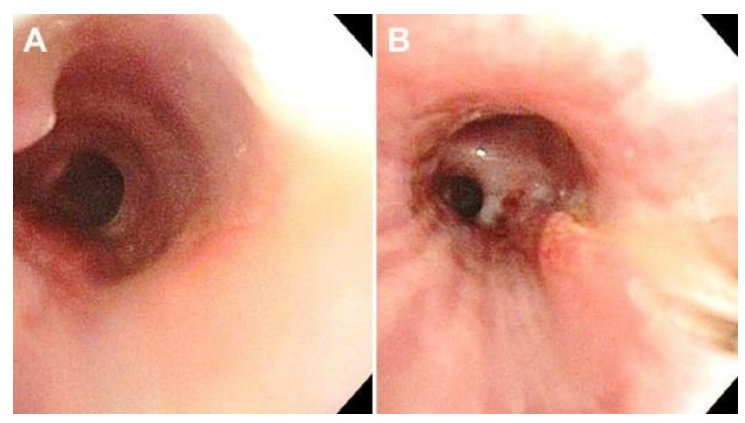

Figure 6. Bronchoscopy for second session of KTP laser therapy 4 months after initial session.

Subsequently image-guided radiotherapy and endobronchial brachytherapy. Were performed. A year later the patient was doing well. Repeat thoracic CT scan showed the left mainstem bronchus to be open (Figure 7).
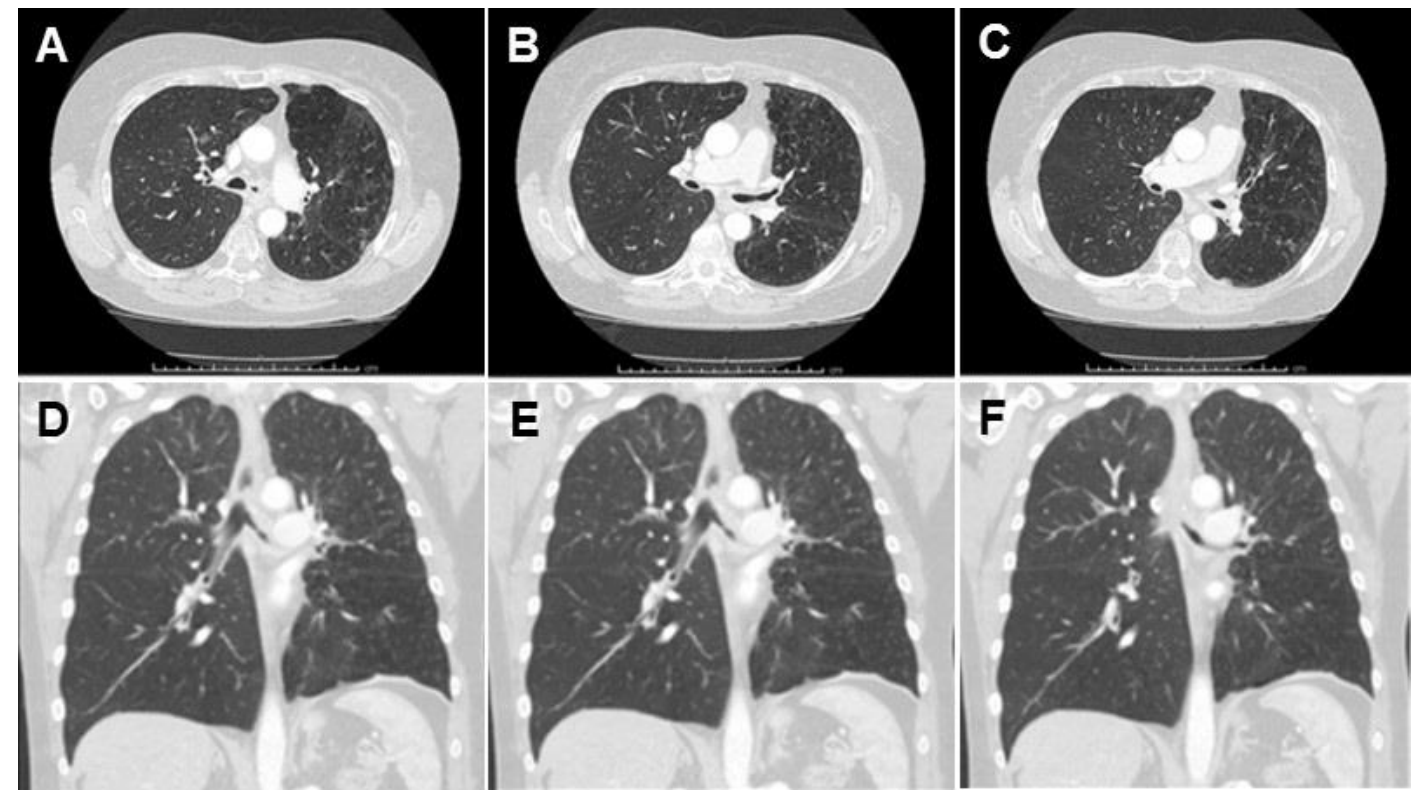

Figure 7. Repeat thoracic CT scan 1 year after laser therapy. A-C: representative axial views in lung windows. D-F: representative coronal views in lung windows.

\section{References}

1. Huo Z, Meng Y, Wu H, Shen J, Bi Y, Luo Y, Cao J, Liang Z. Adenoid cystic carcinoma of the tracheobronchial tree: clinicopathologic and immunohistochemical studies of 21 cases. Int J Clin Exp Pathol. 2014 Oct 15;7(11):7527-35. [PubMed]

2. Iqbal MS, Aslam S. Primary adenoid cystic carcinoma of lung - an overview. Clin Oncol. 2016;1:1158. 